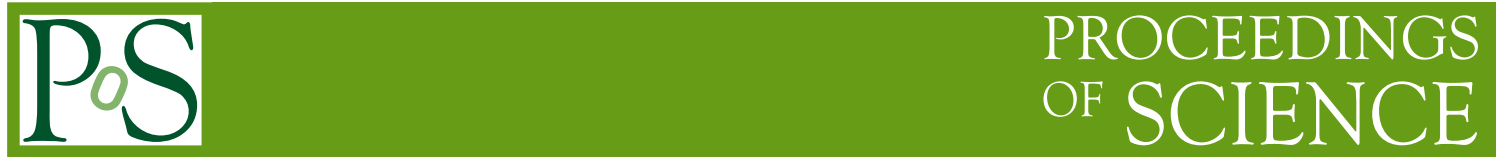

\title{
Swift XRT monitoring of the ULX M33 X8
}

\section{Valentina La Parola*, Giancarlo Cusumano, Teresa Mineo}

INAF - IASF Palermo

E-mail: laparola@ifc.inaf.it, cusumanodifc.inaf.it,mineo@ifc.inaf.it

\section{Antonino D'Aì}

Affiliation

E-mail: antonino.dai@unipa.it

\begin{abstract}
M33 X-8 is the closest example of a persistent ULX and, with luminosity of a few $10^{39} \mathrm{erg} \mathrm{s}^{-1}$, it marks the faint end of the ULX luminosity function. We report here the results of a Swift-XRT 6-year monitoring campaign of this source that extends to 16 years its monitoring in the soft Xray band. We find that M33 X-8 shows a low flux variability and no significant spectral variation along the monitoring. The time-averaged spectrum can be well described by a thermal model, either in the form of a slim disk, or as a combination of a Comptonized corona and a standard accretion disk.
\end{abstract}

Swift: 10 Years of Discovery,

2-5 December 2014

La Sapienza University, Rome, Italy

${ }^{*}$ Speaker. 


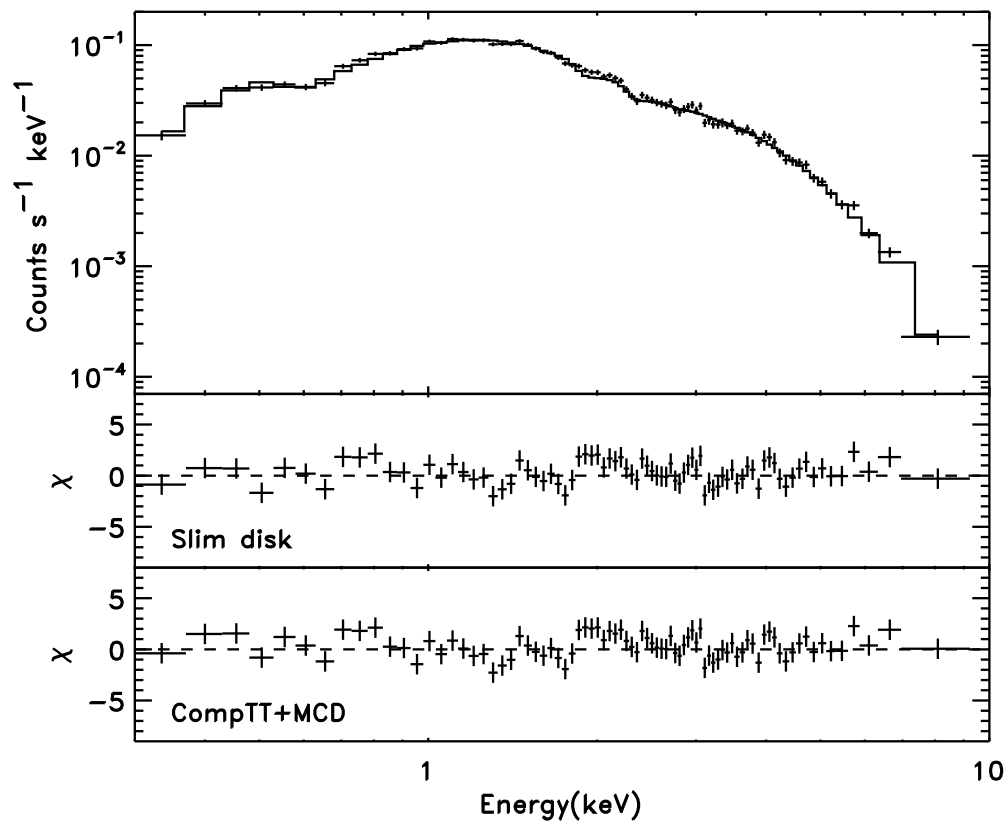

Figure 1: Top panel: data and best fit model (with model DISKPBB) for the average M33 X-8 spectrum. Lower panels: residuals for the best fitting spectral models.

\section{Introduction}

Ultraluminous X-ray sources are point-like, off-nuclear objects observed in many nearby galaxies (e.g. [1, 2]). Their isotropic luminosity between $\sim 10^{39}$ and $\sim 10^{41} \mathrm{erg} \mathrm{s}^{-1}$ in the Xray band, the paucity of information in other energy bands, and their variegated phenomenology [3], led to the formulation of several hypotheses to explain their nature (and indeed they may form an heterogenous class of sources): from the presence of an intermediate mass black hole (IMBH, with $\left.\mathrm{M}_{\mathrm{BH}} \sim 100-1000 \mathrm{M}_{\odot}[4,5]\right)$ in a sub-Eddington accretion regime, to super-critical accretion onto a stellar mass black hole [6] or a beamed/collimated radiation [7, 8].

M33 X-8 [9] is the closest ( $~ 820 \mathrm{kpc},[10])$ example of a persistent ULX, whose X-ray luminosity (a few $10^{39} \mathrm{erg} \mathrm{s}^{-1}$ ) makes it belong to the faint end of the ULX luminosity distribution. Its position is roughly coincident with the center of its host galaxy, but the upper limit derived by [11] on the mass of the nucleus of $\mathrm{M} 33\left(1500 M_{\odot}\right)$ and the detection of a $\sim 106 \mathrm{~d}$ periodical modulation [12] rules out the possibility that the source is a low luminosity AGN. On the other hand no optical counterpart could be identified, due to the extreme crowding of the field.

\section{The Swift-XRT monitoring: analysis and results}

Swift-XRT [13] observed the central region of M33 fifteen times between December 2007 and June 2013, for a total exposure time of $115 \mathrm{ks}$. The source shows no significant spectral variability along the monitoring, so we performed our analysis on the spectrum obtained cumulating the data over the entire monitoring. We tested several models commonly used to describe the spectra of 
ULXs. M33 X-8 is characterized by a spectrum that can be equally well described either by a slim disk model or by a two component disk-corona model.

\subsection{The disk-corona model}

To test the corona+disk scenario, we fitted the spectra using a model consisting of accretion disk and Comptonized emission (DISKBB and COMPTT in XSPEC, respectively). Our results suggest the presence of a cool $(\sim 0.6 \mathrm{keV})$ disk plus a warm (electron temperature $1.3 \mathrm{keV})$ optically thick $(\tau \sim 11)$ corona. This set of values, already observed in this and in other ULX sources (e.g. [6]), breaks the similarity between ULXs and Galactic black hole binaries, where a Comptonized corona over the disk is observed to be hotter $(\mathrm{kT} \gtrsim 50 \mathrm{keV})$ and thinner $(\tau \lesssim 1)$ (e.g. [14]). In the case of ULXs, the strong radiation pressure resulting from a high accretion rate may induce important outflows from the inner part of the disk, resulting in a thick and warm Comptonizing wind that blocks the view to the inner and hottest part of the disk. When the radiation pressure decreases, the wind weakens and its photospheric radius gets smaller, exposing the inner (hotter) part of the disk [15].

\subsection{The slim disk model}

The slim disk hypothesis has been already proposed as a physically consistent description of the spectrum of M33 X-8 by [16], [17] and [18]. In fact, a slim disk, where advective energy transport dominates over radiative cooling $[19,20])$, can be invoked to explain supercritical accretion: in such a disk, a lower radiation pressure allows for a super-critical accretion rate, and can provide a moderate super-Eddington luminosity. This scenario can be modelled with a modified version of the standard accretion disk, with a different slope for the temperature radial dependence [21]. Assuming $\mathrm{T} \propto \mathrm{R}^{-\mathrm{p}}$, a value of $\mathrm{p}=0.75$ indicate a standard disk, expected for sources in a pure thermal state, while a value $\mathrm{p}=0.5$ describes a slim disk. The value of the temperature gradient $\mathrm{p}$ that better fits our data is $0.60 \pm 0.02$ (with a inner temperature of $\sim 1.43 \mathrm{keV}$ ), not consistent with the standard disk value of 0.75 , thus implying that the disk is in an advective regime, with a super-critical accretion rate: according to [21], for a mass of $\sim 10 M_{\odot}$, the observed luminosity and temperature are consistent with a mass accretion rate of a factor of 10 higher than the critical rate.

\section{A 16-year long-term monitoring}

Figure 2 shows the $0.3-10 \mathrm{keV} 16$-year long term light curve of M33 X-8. The observed fluxes for the Swift XRT pointings are derived from using the DISKPBB best fit model for each single observation. We report also the 0.3-10 keV flux observed in the past by SAX [22], Chandra [23, 24], XMM [17], and Suzaku [18]. The source shows a weak flux variability over the entire XRT monitoring (that spans over a time baseline of $\sim 6$ years), with an average $0.3-10 \mathrm{keV}$ flux of $(1.57 \pm 0.02) \times 10^{-11} \mathrm{erg} \mathrm{cm}^{-2} \mathrm{~s}^{-1}$, and variations of $\lesssim 15 \%$ around this value. The average luminosity, assuming isotropic emission at the distance on $820 \mathrm{Mpc}$ is $\sim 1.3 \times 10^{39} \mathrm{erg} \mathrm{s}^{-1}$, and locates it at the low luminosity end of the known ULX sample. The flux variations observed with Swift are consistent with what observed with the other satellites that recorded a luminosity between 1.0 and $1.6 \times 10^{39} \mathrm{erg} \mathrm{s}^{-1}$. Significant long-term flux variability is commonly observed in ULX, 


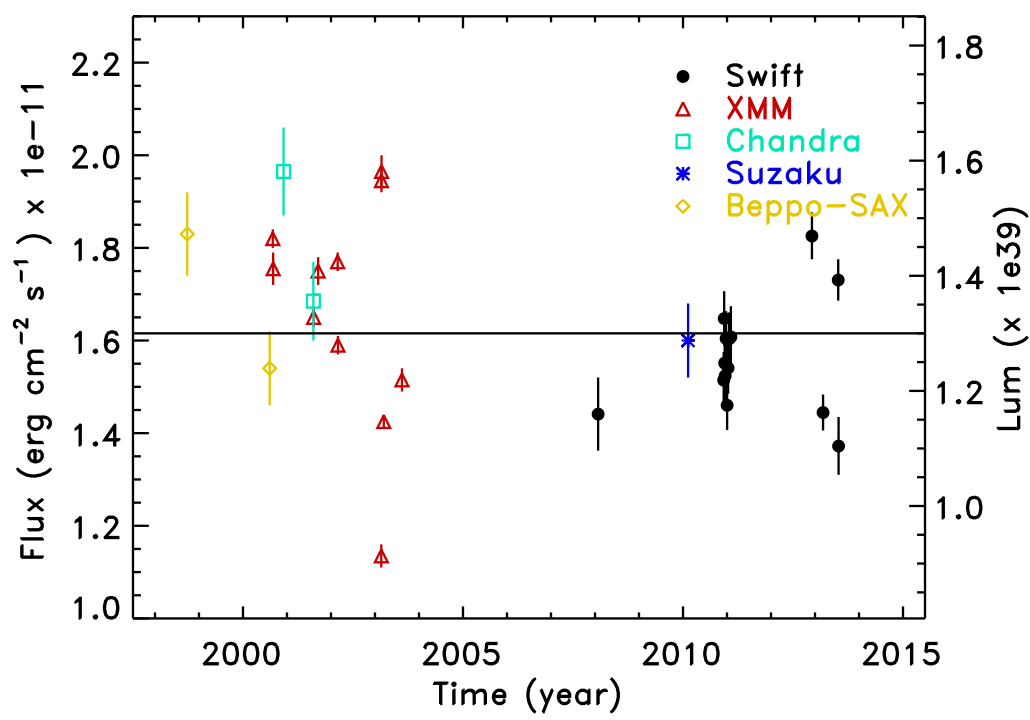

Figure 2: Long term light curve of M33 X-8. Each point corresponds to a single observation. The luminosity on the right axis has been evaluated assuming a distance of $820 \mathrm{kpc}$. We have associated an arbitrary $5 \%$ statistical error to the BeppoSAX, Chandra and Suzaku points. The horizontal line represents the Eddington luminosity for a $10 \mathrm{M}_{\odot}$ black hole.

but the variability amplitude observed in M33 X-8 is lower than that observed in other persistent ULXs, that may reach a factor of $\sim 5$ in flux amplitude, as shown, e.g., by [25].

We have also compared the spectral shape of M33 X-8 as observed in different epochs. To this aim we have used as a probe the spectral parameters obtained from a power law+disk model both for the data analysed in this work and for all the datasets where this information is available in literature: albeit phenomenological, this model has the advantage of having been used to successfully fit the data in all past studies. Thus it can be used as a comparison, although care must be drawn from deriving from this direct physical quantities. Figure 3 shows the value of the disk temperature (right panel) and of the power law slope (left panel) as a function of the observed flux. The result is a substantial invariance of the spectral shape along the history of the source, at least within the sensitivity of the data.

\section{References}

[1] G. Fabbiano, X rays from normal galaxies, ARAA (1989), 27, 87

[2] D. A. Swartz, R. Soria, A. F. Tennant \& M. Yukita, A Complete Sample of Ultraluminous X-ray Source Host Galaxies, ApJ (2011), 741, 49

[3] H. Feng \& R. Soria, Ultraluminous X-ray sources in the Chandra and XMM-Newton era, New A Rev (2011), 55, 166

[4] E. J. M. Colbert \& R. F. Mushotzky, Compact X-ray sources in nearby galaxy nuclei, ApJ (1999), 519, 89 

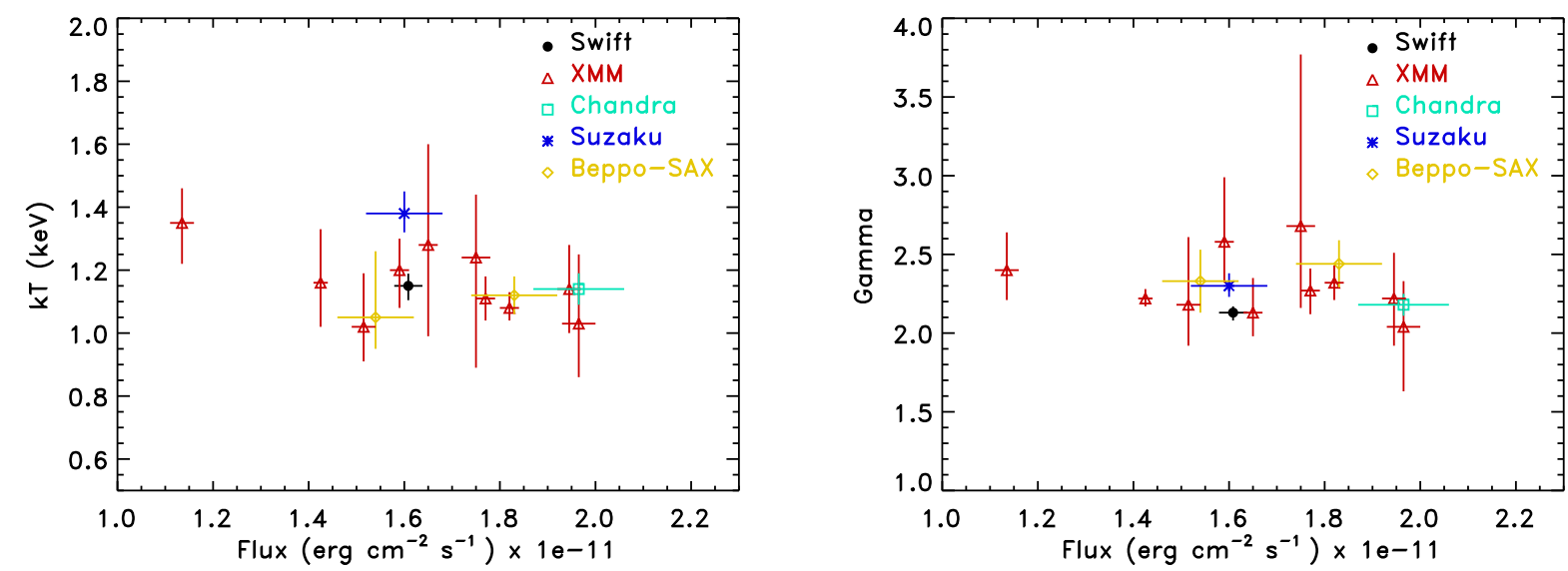

Figure 3: Disk temperature (right panel) and power law index (left panel) vs. 0.3-10 keV flux, for those dataset where this information is available. The temperature and power law slope are derived for all the datasets using a power law+ diskbb model.

[5] A. D. Sutton, T. P. Roberts \& M. J. Middleton, The ultraluminous state revisited: fractional variability and spectral shape as diagnostics of super-Eddington accretion, MNRAS (2013), 435, 1758

[6] J. C. Gladstone, T. P. Roberts \& C. Done, The ultraluminous state, MNRAS (2009), 397, 1836

[7] A. R. King, M. B. Davies, M. J. Ward, G. Fabbiano \& M. Elvis, Ultraluminous X-Ray Sources in External Galaxies, ApJL (2001), 552, L109

[8] A. R. King, Masses, beaming and Eddington ratios in ultraluminous X-ray sources, MNRAS (2009), 393, L41

[9] K. S. Long, S. Dodorico, P. A. Charles, \& M. A. Dopita, Observations of the X-ray sources in the nearby SC galaxy M33, ApJL (1981), 246, L61

[10] W. L. Freedman, B. F. Madore, B. K. Gibson, et al., Final Results from the Hubble Space Telescope Key Project to Measure the Hubble Constant, ApJ (2001), 553, 47

[11] K. Gebhardt, T. R. Lauer, J. Kormendy, et al., M33: A Galaxy with No Supermassive Black Hole, AJ (2001), 122, 2469

[12] G. Dubus, P. A. Charles, K. S. Long, \& P. J. Hakala, A 106 Day Period in the Nuclear Source X-8 in M33, ApJL (1997), 490, L47

[13] D. N. Burrows, J. E. Hill, J. A. Nousek, et al., The Swift X-Ray Telescope, SSRv (2005), 120, 165

[14] A. Kubota, \& C. Done, The very high state accretion disc structure from the Galactic black hole transient XTE J1550 - 564, MNRAS (2004), 353, 980

[15] M. J. Middleton, A. D. Sutton, T. P. Roberts, F. E. Jackson, \& C. Done, The missing link: a low-mass $X$-ray binary in M31 seen as an ultraluminous X-ray source, MNRAS (2012), 420, 2969

[16] S.-S. Weng, J.-X. Wang, W.-M. Gu, J.-F. \& Lu, Spectral Analyses of the Nearest Persistent Ultraluminous X-Ray Source M 33 X-8, PASJ (2009), 61, 1287

[17] M. J. Middleton, A. D. Sutton, \& T. P. Roberts, X-ray spectral evolution in the ultraluminous X-ray source M33 X-8, MNRAS (2011), 417, 464 
[18] N. Isobe, A. Kubota, H. Sato, \& T. Mizuno, Suzaku Investigation into the Nature of the Nearest Ultraluminous X-Ray Source, M33 X-8, PASJ (2012), 64, 119

[19] M. A. Abramowicz, B. Czerny, J. P. Lasota, \& E. Szuszkiewicz, Slim accretion disks, ApJ (1988), 332, 646

[20] K. Ebisawa, P. Życki, A. Kubota, T. Mizuno, \& K.-y. Watarai, Accretion Disk Spectra of the Ultra-luminous X-ray Sources in Nearby Spiral Galaxies and Galactic Superluminal Jet Sources, ApJ (2003), 597, 780

[21] K.-y. Watarai, J. Fukue, M. Takeuchi, S. Mineshige, Galactic Black-Hole Candidates Shining at the Eddington Luminosity, PASJ (2000), 52, 133

[22] A. N. Parmar, L. Sidoli, T. Oosterbroek, P. A. Charles, G. Dubus, M. Guainazzi, P. Hakala, W. Pietsch, G. Trinchieri, BeppoSAX spectroscopy of the luminous X-ray sources in M 33, AAP (2001), 368, 420

[23] V. La Parola, F. Damiani, G. Fabbiano, G. Peres, Spectral Variability of the Nucleus of M33 in a Chandra/ACIS Observation, ApJ (2004), 583, 758

[24] G. Dubus, P. A. Charles, K. S. Long, High resolution Chandra X-ray imaging of the nucleus of M 33, $A \& A$ (2004), 425, 95

[25] F. Pintore, L. Zampieri, A. Wolter, \& T. Belloni, Ultraluminous X-ray sources: a deeper insight into their spectral evolution, MNRAS (2014), 439, 3461 